\title{
Selective excitation and imaging of precessional modes in soft-magnetic squares
}

\author{
Andreas Neudert*, Paul S. Keatley*, Volodymyr V. Kruglyak*, Jeffrey McCord ${ }^{\dagger}$ and Rob J. Hicken* \\ ${ }^{*}$ School of Physics, University of Exeter, Exeter, UK, EX4 4QL \\ Email: a.neudert@exeter.ac.uk \\ ${ }^{\dagger}$ Institute of Solid State and Materials Research, IFW Dresden, 01171 Dresden, Germany
}

\begin{abstract}
We investigated the high-frequency modes of $40 \mu \mathrm{m}$ wide, $160 \mathrm{~nm}$ thick $\mathrm{Fe}_{70} \mathrm{Co}_{8} \mathrm{~B}_{12} \mathrm{Si}_{10}$ squares using time-resolved scanning Kerr microscopy. Two modes were identified using pulsed field excitation while the spatial character of the outof-plane and in-plane magnetization component was investigated using harmonic field excitation. The field dependence of the two modes has been fitted using the Damon-Eshbach model.
\end{abstract}

\section{INTRODUCTION}

The high frequency properties of soft magnetic thin film elements are essential to the operation of a variety of rf devices and magnetic field sensors. The response of the magnetization to high frequency magnetic fields should normally be fast and homogeneous if optimum device performance is to be achieved. However patterning modifies the effective magnetic field within an element and determines the spectrum of dynamical modes that may be excited. This mode spectrum must be characterized and fully understood if the high frequency magnetic response is to be controlled. A number of theoretical and experimental publications addressed this problem in recent years [1-13]. In this paper we report on how time-resolved scanning Kerr microscopy has been used to understand the precessional mode spectrum in soft-magnetic square elements.

In Refs. [12], [13], the mode spectrum of square $\mathrm{Ni}_{81} \mathrm{Fe}_{19}$ elements of comparable size was investigated using static Kerr microscopy. There the excitation field was localized in the center of the element and had in-plane and out-of-plane components. In our experiment the excitation field is spatially homogeneous and lies in the plane. Furthermore we use a timeand vector-resolved setup, allowing us to image the temporal evolution of the excited modes.

\section{EXPERIMENT}

The ferromagnetic amorphous film was deposited by magnetron sputtering onto a transparent glass substrate and structured by ion beam etching. The sample consisted of squares of $\mathrm{Fe}_{70} \mathrm{Co}_{8} \mathrm{~B}_{12} \mathrm{Si}_{10}$ with width of $40 \mu \mathrm{m}$ and thickness of $160 \mathrm{~nm}$ arranged into a square lattice with $10 \mu$ m edge-to-edge separation. Sample deposition was performed in a magnetic field so as to induce a uniaxial magnetic anisotropy parallel to one of the edges of the squares.

Measurements of the high-frequency dynamics were performed with a time-resolved scanning Kerr microscope setup, shown in Fig. 1. A quadrant photodiode bridge detector was used to measure all three components of the magnetization vector simultaneously. The magnetization was probed with 100 fs pulses from a Ti:Sapphire femtosecond laser system running at $80 \mathrm{MHz}$ repetition rate and at a wavelength of $800 \mathrm{~nm}$. The magnetization was excited by the highfrequency magnetic fields generated around the $80 \mu \mathrm{m}$ wide center conductor of a coplanar waveguide fed with pulsed or sinusoidal current waveforms from a pulse or microwave generator, respectively. The pulse and microwave generators were phase locked to the laser so that stroboscopic imaging could be performed. The output frequency of the microwave generator is therefore required to be a multiple of the $80 \mathrm{MHz}$ laser repetition rate. We used two microwave generators for this purpose. The first microwave generator produced the $\mathrm{rf}$ excitation field of $8.0 \mathrm{GHz}$ or $9.2 \mathrm{GHz}$, while a second generator was needed to convert the $10 \mathrm{MHz}$ reference output of the first generator to the $80 \mathrm{MHz}$ signal used to trigger the laser system. This setup gave a phase drift between pump and probe of less than $10 \%$ of a cycle $(\pi / 5)$ within the scanning time of several hours for the time-resolved images.

The sample was placed face down on the waveguide with the laser spot focused on the square element through the glass substrate so that the exciting field was maximised. A polarizing beamsplitter and quadrant photodiodes were used to detect all three components of the magnetization vector simultaneously as described in Ref. [14]. Audio frequency modulation of the pump waveform and phase sensitive detection were used to enhance the sensitivity of the apparatus. To acquire data at different time delays during the excitation an optical delay line with a maximum delay of $8 \mathrm{~ns}$ was used.

The polar Kerr rotation (proportional to the out-of-plane magnetization) in the center of the element was detected for the pulsed field excitation at different external bias field strengths between 50 Oe and 500 Oe. For lower field values the magnetization in the sample began to form a domain structure. By performing a Fast Fourier Transformation (FFT) the spectra of the dynamic excitation were obtained. These spectra are shown in Fig. 2. For every field step we identify two resonant modes that overlap and form a dominant peak with an additional shoulder. In contrast to Tamaru et al. [12] no increase in the number of modes with changing external bias field is found.

To gain insight into the spatial character of the two modes we replaced the pulse generator with a microwave generator to excite the sample with harmonic fields at the resonance 
a)

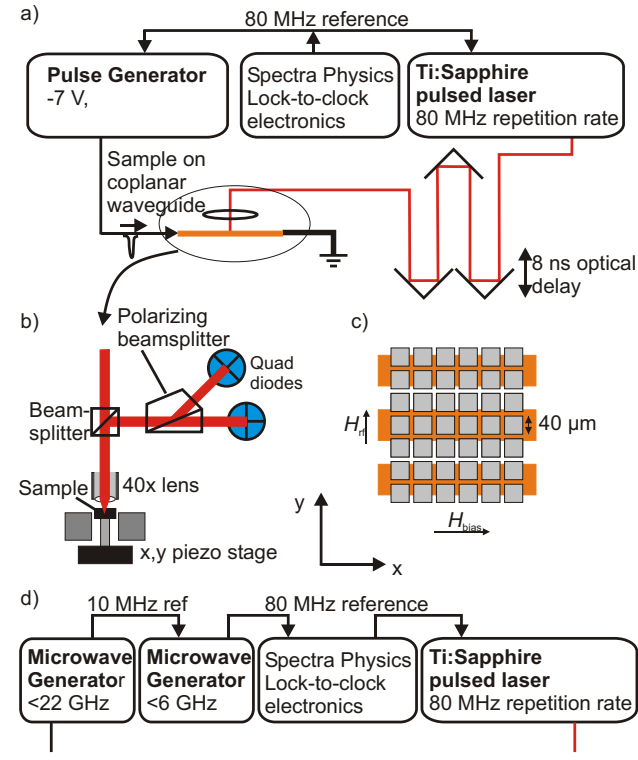

Fig. 1. (a) Experimental setup showing the laser system, synchronizing clock, optical delay line, and pulse generator. The optical detection of the Kerr rotation is shown in (b). The sample is placed face-down on a coplanar waveguide (c). For the harmonic excitation the pulse generator is replaced by a microwave generator and a second microwave generator is needed to phase lock the first microwave generator to the laser.

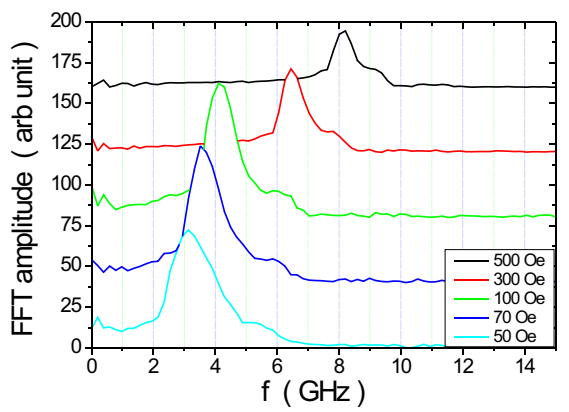

Fig. 2. Spectra aqcuired for different bias field strengths. Two modes can be identified in each spectrum which increase with increasing bias field.

frequency. The measurements were performed at 500 Oe bias field for which the resonance frequencies of the two modes were found to be 8.0 and $9.2 \mathrm{GHz}$, respectively. The dynamic out-of-plane and in-plane magnetization (perpendicular to the bias field, and perpendicular to the static magnetization $\vec{M}$ ) are shown in Fig. 3 for different time delays. In both cases a phase difference between the center and the left and right regions in the element can be seen. The magnetization at the edge of the element lags behind that at the centre.

The images of the $8.0 \mathrm{GHz}$ excitation shows a spatially more homogeneous dynamic response than that of the $9.2 \mathrm{GHz}$ mode. The magnetization at the edges of the element perpendicular to the static magnetization changes less than in the center. The magnetic contrast of the images showing the inplane dynamic magnetization is reduced in comparison to the out-of-plane images because of the smaller sensitivity to the

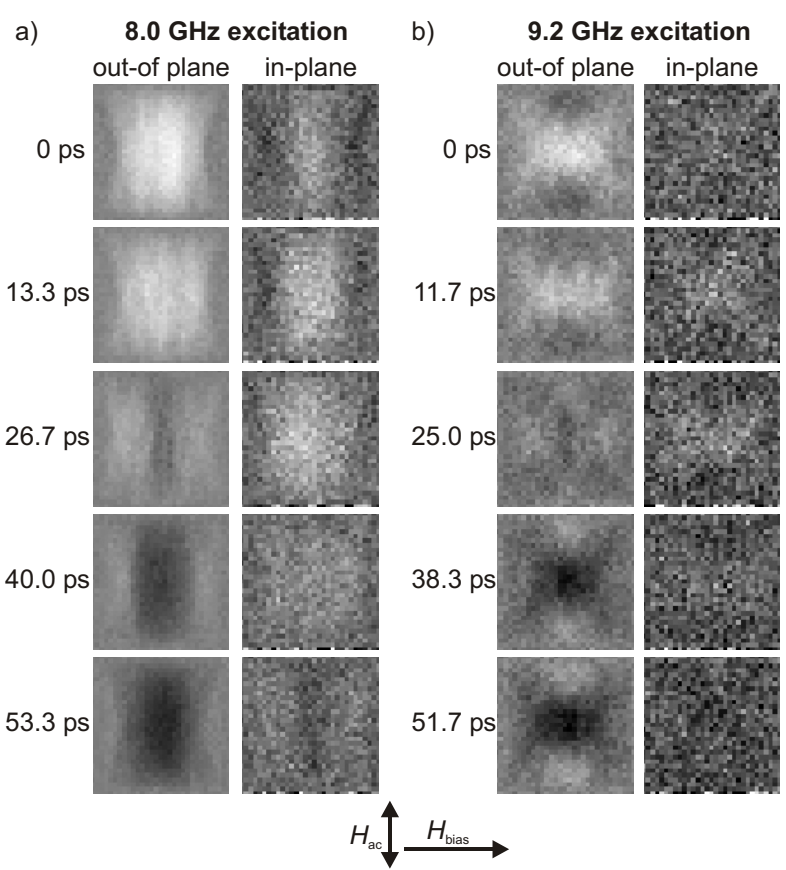

Fig. 3. Time-resolved images of the two modes at 500 Oe bias field. The sample was pumped with a sinusoidal magnetic field at $8.0 \mathrm{GHz}$ (a) and $9.2 \mathrm{GHz}(\mathrm{b})$, respectively. For both modes the out-of-plane component and the in-plane component perpendicular to the static magnetization direction are shown.

longitudinal Kerr contrast. By measuring the components of both Kerr geometries for varying $\mathrm{x}$-positions $(\mathrm{y}=20 \mu \mathrm{m})$ the magnetization orbits at those positions have been determined. Those orbits are shown in Fig. 5. Although the absolute orbit shape can not be determined, because of the different dependence of the longitudinal and polar Kerr effect upon the in and out of plane magnetization component, it can be seen that the relative shape does not change significantly with distance from the edge of the element. The phase difference of the magnetization between the center and the edge of the element is the reason for the different starting position of the magnetization trajectory. The cone angle of the magnetization is largest in the center of the element and decreases towards the edge.

The response to the higher frequency excitation is more spatially inhomogeneous. The magnetization shows a maximum in the center of the element and a change of sign in the regions close to the top and bottom edge. This is also shown in Fig. 4 with two linescans across the center of the image taken at $0 \mathrm{ps}$ for each mode. The linescan along the static magnetization direction shows a positive out-of-plane magnetization direction along the whole width of the element, whereas the linescan along the excitation field (perpendicular to static $\vec{M}$ ) displays a change of sign close to the edges of the element.

The linescans perpendicular to the static magnetization direction were fitted with to the form

$$
y=A_{1} \sin \left(2 \pi x / \lambda_{1}\right)+A_{2} \sin \left(2 \pi x / \lambda_{2}\right)
$$




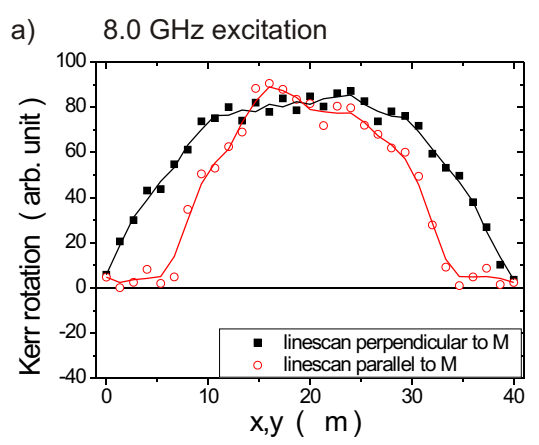

b) $\quad 9.2 \mathrm{GHz}$ excitation

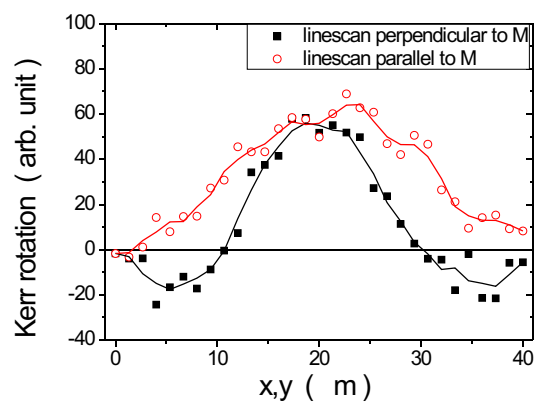

Fig. 4. Linescan through the center of the out-of-plane image of the $8.0 \mathrm{GHz}$ mode (a) and $9.2 \mathrm{GHz}$ mode (b) at $0 \mathrm{ps}$. There is a clear change of sign visible for the dynamic magnetization of the $9.2 \mathrm{GHz}$ mode along the y-axis. The lines are a 3 point average of the data points. The same scaling of the data has been applied throughout the paper.

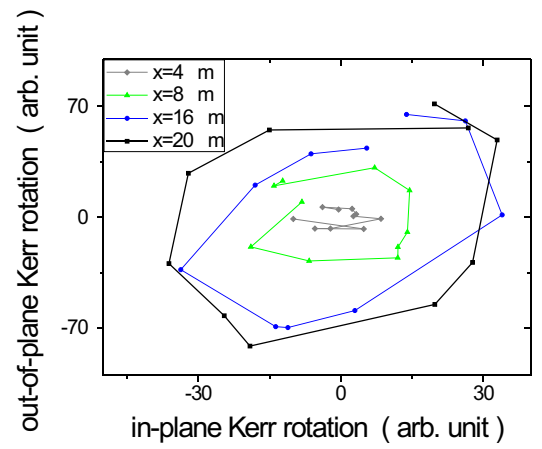

Fig. 5. Orbit of the $8 \mathrm{GHz}$ mode at different positions $(\mathrm{x}=4,8,16,20 \mu \mathrm{m}$; $\mathrm{y}=20 \mu \mathrm{m})$

as shown in Fig. 6. The amplitudes for the $8.0 \mathrm{GHz}$ linescan are $A_{1}=83.4$ and $A_{2}=7.73$ giving a ratio of 0.09 between the higher and lower wavelength mode. In the $9.2 \mathrm{GHz}$ linescan the amplitudes are almost equal, $A_{1}=29.5$ and $A_{2}=-29.3$, but with a different sign as already mentioned. The wavelengths are almost identical in the two linescans: $\lambda_{1}=79.8 \mu \mathrm{m}(8.0 \mathrm{GHz}$ linescan $)$ and $\lambda_{1}=79.5 \mu \mathrm{m}$ (9.2 GHz linescan), and $\lambda_{2}=26.8 \mu \mathrm{m}(8.0 \mathrm{GHz}$ linescan $)$ and $\lambda_{2}=25.8 \mu \mathrm{m}(9.2 \mathrm{GHz}$ linescan $)$, respectively. The similarity of the amplitudes of the two modes if the sample is excited with the $9.2 \mathrm{GHz}$ frequency is also visible in Fig. 7 showing a Lorentzian fit decomposed into the two individual Lorentzian functions. This means that for the excitation of the a)

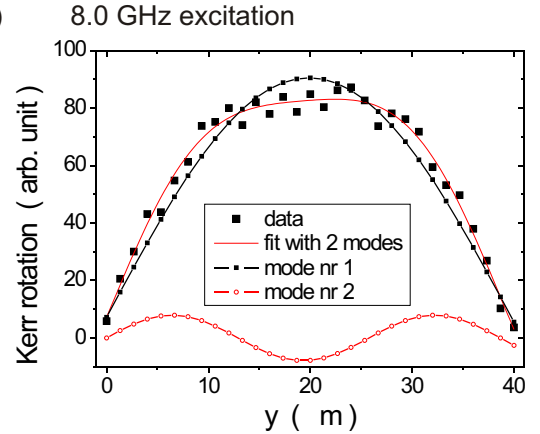

b)

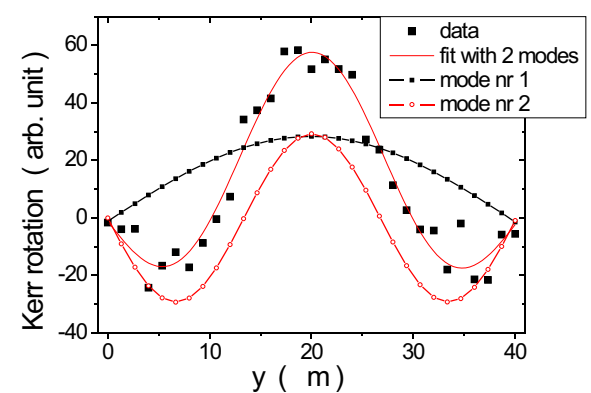

Fig. 6. Linescan across the out-of-plane image of the $8.0 \mathrm{GHz}$ mode (a) and $9.2 \mathrm{GHz}$ mode (b) at $0 \mathrm{ps}$ and a fit using sine functions with two different wavelengths. The individual sine curves determined from the fit are also displayed.

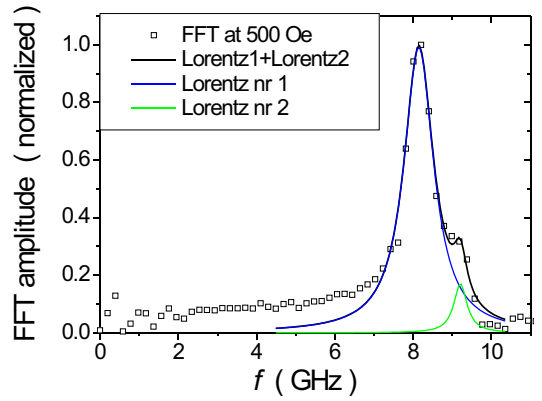

Fig. 7. Fit of the spectrum at 500 Oe to two Lorentzian functions.

magnetization at $9.2 \mathrm{GHz}$ both modes are excited due to the finite linewidth of the modes and the spatial character of both modes can be seen in the time-resolved images.

The spatial distribution of each mode along the axis parallel to the excitation field can be described by a standing wave. The wavelengths are of the order of the size of the element so that the waves have a magnetostatic character. This type of magnetostatic wave is typical of a Damon-Eshbach type mode [15]. The frequencies of those modes are described by the following formula:

$$
f=\frac{\gamma}{2 \pi} \sqrt{H\left(H+4 \pi M_{s}\right)+\left(2 \pi M_{s}\right)^{2}\left(1-e^{-2 k d}\right)}
$$

with gyromagnetic ratio $\gamma$, magnetic field $H$, saturation magnetization $M_{s}$, wave number $k$, and thickness $d$. The wave 


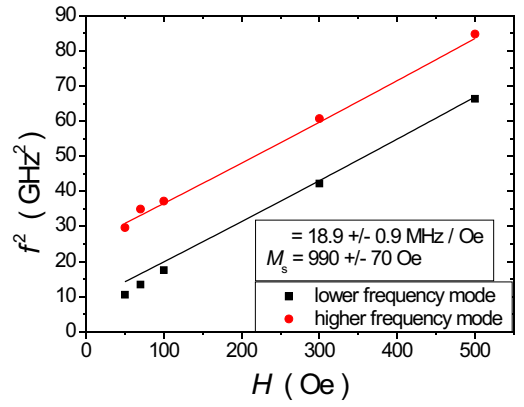

Fig. 8. Frequencies of the two modes vs. applied field. Fitting of this data with a Damon-Eshbach model yields $M_{s}=990 \pm 70$ Oe and $\gamma=18.9 \pm 0.9 \mathrm{MHz} / \mathrm{Oe}$ for mode numbers $\mathrm{n}=1,3$.

number depends on the mode number $n$ as:

$$
k=n \pi / w
$$

with the width $w$ of the squares equal to $40 \mu \mathrm{m}$. Since the excitation fields (pulsed and harmonic) in our experiment are spatially homogeneous only odd-numbered modes are expected to couple to them. The frequencies of the two peaks were determined by fitting the spectra with two Lorentzian functions and are shown in Fig 8. The two modes were fitted simultaneously using eq. 2 with fixed mode numbers of 1 and 3. The fit is shown in the figure and the resulting values for $M_{s}$ and $\gamma$ are: $M_{s}=990 \pm 70$ Oe and $\gamma=18.9 \pm 0.9 \mathrm{MHz} / \mathrm{Oe}$. The wavelength $\lambda=2 \pi / k=2 w / n$ of those two modes is $80 \mu \mathrm{m}$ for the lower frequency mode and $26.7 \mu \mathrm{m}$ for the higher frequency mode. Those wavelengths are in good agreement with those deduced from the linescans shown in Fig. 6.

\section{CONCLUSION}

In conclusion we have demonstrated how the spatial character of spin wave modes in patterned microscale elements can be investigated using scanning Kerr microscopy with phase-locked harmonic excitation. At fields above the domain nucleation value two modes could be distinguished. The lower frequency excitation resulted in a spatially rather homogeneous response of the magnetization with decreasing dynamical magnetization amplitude at the edges, whereas the response to the higher frequency excitation displayed a standing wave pattern along the excitation field axis that is a superposition of the $n=1$ and $n=3$ DE modes of the element. By detecting the out-of-plane and in-plane Kerr rotation the relative shape of the magnetization orbit was investigated. It was found that the orbit does not change its relative shape at different positions relative to the edge of the element. The variation of the frequencies of both modes with increasing bias field was well described by the Damon-Eshbach model.

\section{REFERENCES}

[1] B. C. Choi, M. Belov, W. K. Hiebert, G. E. Ballentine, and M. R. Freeman, "Ultrafast magnetization reversal dynamics investigated by time domain imaging," Physical Review Letters, vol. 86, p. 728, 2001.
[2] A. Barman, V. V. Kruglyak, R. J. Hicken, A. Kundrotaite, and M. Rahman, "Anisotropy, damping, and coherence of magnetization dynamics in a $10 \mu \mathrm{m}$ square $\mathrm{ni}_{81} \mathrm{fe}_{19}$ element," Applied Physics Letters, vol. 82, p. 3065, 2003.

[3] A. Barman, V. V. Kruglyak, R. J. Hicken, J. M. Rowe, A. Kundrotaite, J. Scott, and M. Rahman, "Imaging the dephasing of spin wave modes in square thin film magnetic element," Physical Review B, vol. 69, p. 174426, 2004.

[4] C. H. Back, D. Pescia, and M. Buess, "Vortex dynamics," Topics in Applied Physics, vol. 101, p. 137, 2006.

[5] C. Bayer, J. Jorzick, S. O. Demokritov, A. N. Slavin, K. Y. Guslienko, D. V. Berkov, N. L. Gorn, M. P. Kostylev, and B. Hillebrands, "Spinwave excitations in finite rectangular elements," Topics in Applied Physics, vol. 101, p. 57, 2006.

[6] M. Bailleul, R. Höllinger, and C. Fermon, "Microwave spectrum of square permalloy dots: Quasisaturated state," Physical Review B, vol. 73, p. 104424, 2006.

[7] M. Bailleul, R. Höllinger, K. Perzlmaier, and C. Fermon, "Microwave spectrum of square permalloy dots: Multidomain state," Physical Review $B$, vol. 76, p. 224401, 2007.

[8] B. C. Choi, J. Rudge, M. R. Freeman, Y. K. Hong, and Q. F. Xiao, "Nonequilibrium magnetic domain structures as a function of speed of switching process in $\mathrm{ni}_{80} \mathrm{fe}_{20}$ thin-film element," IEEE Transactions on Magnetics, vol. 43, p. 2, 2007.

[9] F. Giesen, J. Podbielski, B. Botters, and Gründler, "Vortex circulation control in large arrays of asymmetric magnetic rings," Physical Review $B$, vol. 75, p. 184428, 2007.

[10] K. Y. Guslienko, R. W. Chantrell, and A. N. Slavin, "Dipolar localization of quantized spin-wave modes in thin rectangular magnetic elements," Physical Review B, vol. 68, p. 024422, 2003.

[11] A. Neudert, J. McCord, D. Chumakov, R. Schäfer, and L. Schultz, "Small-amplitude magnetization dynamics in permalloy elements investigated by time-resolved wide-field kerr microscopy," Physical Review $B$, vol. 71, p. 134405, 2005.

[12] S. Tamaru, J. A. Bain, R. J. M. van de Veerdonk, T. M. Crawford, M. Covington, and M. H. Kryder, "Imaging of quantized magnetostatic modes using spatially resolved ferromagnetic resonance," Journal of Applied Physics, vol. 91, p. 8034, 2002.

[13] S. Tamaru, J. A. Bain, R. J. M. van de Veerdonk, T. M. Crawford, M. Covington, and M. H. Kryder, "Measurement of magnetostatic mode excitation and relaxation in permalloy films using scanning kerr imaging," Physical Review B, vol. 70, p. 104416, 2004.

[14] P. S. Keatley, V. V. Kruglyak, R. J. Hicken, J. R. Childress, and J. A. Katine, "Acquisition of vector hysteresis loops from micro-arrays of nano-magnets," Journal of Magnetism and Magnetic Materials, vol. 306, pp. 298-301, 2006.

[15] R. W. Damon and J. R. Eshbach, "Magnetostatic modes of a ferromagnetic slab," Journal of Applied Physics, vol. 31, no. 5, pp. S104-S105, 1960. 